\title{
Relative Contributions of Intraoperative Low Dose Ketamine, Lidocaine and Ketamine-Lidocaine Combination in Addition to Intrathecal Morphine for Postoperative Analgesia in Open Liver Resection: A Prospective, Randomized, Four-Arm, Triple Blind, Placebo-Controlled Trial
}

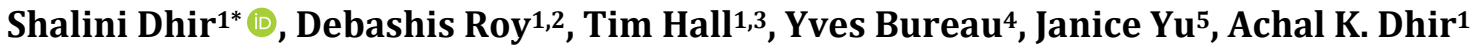 \\ 1Department of Anesthesia and Perioperative Medicine, Schulich School of Medicine, London, Ontario, Canada \\ ${ }^{2}$ Sunnybrook Health Sciences Centre, Department of Anesthesiology, Toronto, Ontario, Canada \\ ${ }^{3}$ Department of Anesthesia \& Perioperative Medicine, Auckland Hospital, University of Auckland, Auckland, New Zealand \\ ${ }^{4}$ Department of Psychology, Department of Medical Biophysics, Western University, London, Ontario, Canada \\ ${ }^{5}$ Department of Anesthesia, Michael G. DeGroote School of Medicine, McMaster University, Hamilton, Ontario, Canada \\ Email: *sdhir2@uwo.ca
}

How to cite this paper: Dhir, S., Roy, D., Hall, T., Bureau, Y., Yu, J. and Dhir, A.K. (2020) Relative Contributions of Intraoperative Low Dose Ketamine, Lidocaine and Ketamine-Lidocaine Combination in Addition to Intrathecal Morphine for Postoperative Analgesia in Open Liver Resection: A Prospective, Randomized, Four-Arm, Triple Blind, Placebo-Controlled Trial. Open Journal of Anesthesiology, 10, 313-326. https://doi.org/10.4236/ojanes.2020.109028

Received: July 29, 2020

Accepted: September 24, 2020

Published: September 27, 2020

Copyright $\odot 2020$ by author(s) and Scientific Research Publishing Inc. This work is licensed under the Creative Commons Attribution International License (CC BY 4.0).

http://creativecommons.org/licenses/by/4.0/

\section{Abstract}

Background and Aims: Open liver resection requiring an upper abdominal incision is associated with significant opioid use due to postoperative pain. We tested the hypothesis that the intraoperative combination of low dose lidocaine and ketamine would reduce opioid consumption when given in conjunction with intrathecal morphine for liver resection surgery. Methods: In this triple blind, parallel group four-arm placebo-controlled trial, we randomized 124 adult ASA 2 - 4 liver resection patients to receive intraoperative lidocaine $0.33 \mathrm{mg} / \mathrm{kg} / \mathrm{h}$ (group L), ketamine $70 \mu \mathrm{g} / \mathrm{kg} / \mathrm{h}$ (group K), combination of lidocaine $0.33 \mathrm{mg} / \mathrm{kg} / \mathrm{h}$ and ketamine $70 \mu \mathrm{g} / \mathrm{kg} / \mathrm{h}$ (group KL) and saline (group P). All patients received $300 \mu \mathrm{g}$ intrathecal morphine prior to induction of anesthesia. All infusions were started immediately after intubation and continued until the end of surgery. Primary outcome measurements included opioid consumption at 24-hours. Secondary outcomes included pain scores, opioid consumption at 48 and 72 -hours and side effects including nausea, vomiting, dizziness, hallucinations, headaches and signs of local anaesthetic toxicity. Patients were followed up for 12 weeks. Results: There was 
no difference in the primary outcome of opioid consumption within all 4 groups at rest or movement at 24-hours. Secondary outcome of 48-hour rest pain score was significantly higher in the $\mathrm{L}$ group $(\mathrm{p}=0.03)$ but without any difference in opioid use. There was no difference in any other outcomes between the groups at any time points. Conclusions: Low dose lidocaine and its combination with ketamine did not add any analgesic/morphine sparing benefit in the presence of single dose intrathecal morphine in patients with major liver resection.

\section{Keywords}

Lidocaine, Ketamine, Intrathecal, Morphine, Analgesia, Liver Resection

\section{Introduction}

Major liver resection involving bilateral subcostal rooftop incision is associated with significant postoperative pain. Intravenous opioids though simple and easy to administer, have clinically relevant dose related side effects and may be associated with inadequate analgesia. It is well known that a functioning epidural provides superior analgesia for major abdominal surgeries, when compared to systemic opioid based analgesia. However, its use is controversial in major liver resection due to possible coagulation abnormalities and may not always be the best analgesic option in this subset of patients [1]. Single dose intrathecal morphine (ITM) is a safe and effective means of providing perioperative analgesia but may not always provide adequate pain relief and may be associated with side effects [2]. Intravenous opioids are the standard of care for the management of postoperative pain in patients who undergo major surgical procedures affecting enteral/oral intake (recommendation\#12) [3]. Non-opioid drugs such as acetaminophen and non-steroidal anti-inflammatory drugs (NSAIDs) may provide insufficient analgesia. Infusion of lidocaine and ketamine are being used for perioperative analgesia and both have shown to reduce postoperative pain and opioid consumption but there is limited evidence supporting the perioperative use of these drugs in combination [4] [5].

The primary hypothesis for this prospective, randomized, triple blind, parallel group, placebo-controlled study was that the addition of intraoperative combination of low dose lidocaine and ketamine would reduce opioid consumption at 24-hours when given in conjunction with intrathecal morphine for liver resection surgery. The primary objective of the study was to measure 24 hours opioid consumption. Secondary objectives included pain scores, opioid consumption at 48 and 72-hours and side effects including nausea, vomiting, dizziness, hallucinations, headaches and signs of local anaesthetic toxicity. We also examined the efficacy of the components of the infusion separately (lidocaine infusion alone and ketamine infusion alone) to see if they had different effects on the opioid consumption. 


\section{Patients and Methods}

After institutional ethics approval and written informed consent, we enrolled 124 adult ASA physical status II-IV patients aged 18 - 80 years scheduled for elective major liver resection due to malignancy. At the time of ethics approval, public registration of the trial was voluntary therefore it was registered retrospectively at clinicaltrials.gov. Exclusion criteria included inability to use patient-controlled analgesia (PCA) or the verbal rating scale (VRS), language barrier, severe coagulopathy, allergy to study drugs, chronic pain, opioid dependence, alcohol or substance abuse, inoperable condition, inability to perform neuraxial procedure, intensive care admission and/or tracheal extubation not done after surgery. After written, informed consent, patients were instructed on the use of PCA device, educated regarding VRS pain (11-point scale ranging from $0=$ no pain to $10=$ worst possible pain), VRS satisfaction $(0=$ completely unsatisfied, $10=$ very satisfied) and signs and symptoms of local anaesthetic toxicity. Our pharmacy confirmed that the lidocaine was compatible and stable with ketamine.

On the day of surgery, all consented patients scheduled for elective major liver resection were randomly assigned to one of four groups, each with 31 subjects. Random allocation sequence was generated by investigators (SD, JY) using web-based Randomness and Integrity Services (Random.org, Dublin). Patients were enrolled by one of the co-investigators ( $\mathrm{DR}, \mathrm{TH}, \mathrm{AD}$ ). Concealed group allocations in opaque envelopes were given to a person not involved in the study who prepared the drugs according to body weight and handed over to the operating room anaesthesiologist (DR, TH, $\mathrm{AD}$ ). The study was a triple blind design in an effort to reduce any bias in the data collection. However, the randomization sequence was available if an emergent situation arose. The patient, the anaesthesiologist, the surgeon, the acute pain service team (APS), the investigators collecting data and statistician analysing the data were unaware of the group allocation.

After standard monitoring, all patients received ITM (300 $\mu \mathrm{g}$ with $1 \mathrm{ml}$ normal saline as a vehicle) at L2-3 or L3-4 interspace via 25 G Whitacre needle in a sitting position. Anesthesia was induced with midazolam, fentanyl, propofol and rocuronium with doses at the discretion of the operating room anaesthesiologist and maintained with inhalational agent (sevoflurane/desflurane) in air-oxygen mixture with boluses of fentanyl or hydromorphone as required. A right internal jugular catheter was placed after patient was asleep and the drug infusion started. The infusion was stopped 30 minutes prior to the completion of surgery. We decided that patients who did not receive ITM and those who were not extubated postoperatively would be excluded from the study as they would not have received the complete intervention or would be unable to use a PCA device and provide the pain score.

The 4 study groups received the following interventions: Group L received lidocaine infusion at $0.33 \mathrm{mg} / \mathrm{kg} / \mathrm{hr}$, group $\mathrm{K}$ received ketamine infusion at 70 
$\mu \mathrm{g} / \mathrm{kg} / \mathrm{hr}$, group $\mathrm{KL}$ received lidocaine infusion at $0.33 \mathrm{mg} / \mathrm{kg} / \mathrm{hr}$ plus a ketamine infusion at $70 \mu \mathrm{g} / \mathrm{kg} / \mathrm{hr}$. The control group P received a normal saline infusion. Drugs were constituted in a way that the infusion was running at $5 \mathrm{ml} / \mathrm{hr}$ for all groups. The study protocols are in Figure 1.

All patients were in a high dependency care unit postoperatively as is the routine practice for all liver resections in our centre. VRS pain was taken both at rest and with movement and/or deep breathing. The 24-hour count started from the time of ITM.

APS reviewed the patients twice daily and managed the postoperative pain following a standard analgesic regime. A PCA with hydromorphone $0.2-0.3 \mathrm{mg}$ bolus, with a lockout interval of 6 minutes and no background infusion was started for all the patients. Once allowed orally and at the discretion of the APS team, analgesics were switched to oral opioids, as needed. All patients received acetaminophen $650 \mathrm{mg}$ every $6 \mathrm{~h}$ for four days. Post-operatively, all patients were monitored and questioned regarding the presence and severity of study drugs related side effects and satisfaction with pain management.

Opioid consumption for the first, second and third postoperative day (POD) was recorded from the chart maintained by APS team who were blinded to the patient's assigned group. All other data was collected by the study personnel. The data were collected before surgery, at 24,48 and 72 hours after surgery (POD 1, 2 and 3) while they were in the hospital. At 6 and 12 weeks, data were collected by telephone.

The study commenced after institutional ethics approval and written informed consent was taken prior to enrolment. All procedures done in the study followed the ethical guidelines of declaration of Helsinki. Applicable Equator guidelines were followed and the CONSORT 2010 and its extension for reporting

$$
\begin{gathered}
\text { Group L } \\
\mathrm{n}=31
\end{gathered}
$$

Lidocaine $0.33 \mathrm{mg} \mathrm{kg}^{-1} \mathrm{~h}^{-1}$

$138.6 \mathrm{mg}$ lidocaine diluted in $30 \mathrm{ml}$ saline, run at $5 \mathrm{mlh}^{-1}$

Group $\mathrm{K}$
$\mathrm{n}=31$
Ketamine $70 \mu \mathrm{g} \mathrm{kg}^{-1} \mathrm{~h}^{-1}$
$29.4 \mathrm{mg}$ ketamine diluted in $30 \mathrm{ml}$ saline, run at $5 \mathrm{ml} \mathrm{h}^{-1}$

Group KL
$\mathrm{n}=31$
$\begin{gathered}\text { Lidocaine } 0.33 \mathrm{mgkg}^{-1} \mathrm{~h}^{-1}+\text { Ketamine } 70 \mu \mathrm{g} \mathrm{kg}^{-1} \mathrm{~h}^{-1} \\ 29.4 \mathrm{mg} \text { ketamine+138.6 mg lidocaine in } 30 \mathrm{ml} \mathrm{saline} \text {, run at } 5 \mathrm{mlh}^{-1}\end{gathered}$
$\begin{gathered}\text { Group S } \\ \mathrm{n}=31 \\ 30 \mathrm{ml} \text { saline, run at } 5 \mathrm{~mL} \mathrm{~h}^{-1}\end{gathered}$

Figure 1 . The 4 study protocols for a $70-\mathrm{kg}$ subject. 
multi-arm trial parallel group trial were met.

\subsection{Outcomes}

The primary outcome measure of the study was opioid consumption at 24 hours or POD1. Secondary outcome measures included opioid consumption POD 2 and 3, pain severity measured at rest and with activity/deep breathing by VRS and satisfaction with pain management (11-point scale ranging from $0=$ completely dis-satisfied to $10=$ extremely satisfied). Nausea/vomiting and pruritus were assessed using a categorical scale $(0=$ none, $1=$ mild, $2=$ moderate, $3=$ severe), sedation was assessed using a categorical scale $(0=$ awake and alert, $1=$ quietly awake, 2 = asleep but easily aroused, 3 = deep sleep). Presence and severity of side effects related to lidocaine (light-headedness, perioral numbness) and ketamine (disturbed dreams and hallucinations) on POD 1 were recorded using a categorical scale $(0=$ none, $1=$ mild, $2=$ moderate, $3=$ severe $)$. Total non-opioid analgesic, anti-emetic, and anti-pruritic consumption at day 1, 2 and 3 postoperatively were recorded. Pain scores at rest and with movement were also recorded at 6 weeks and 12 weeks post-operatively. Demographics, ASA physical status, anthropometric measurements (height, weight, BMI) were recorded. All opioid analgesics used were converted to intravenous morphine equivalents using https://opioidcalculator.practicalpainmanagement.com/conversion.php.

We hypothesized that the addition of low doses of intraoperative intravenous lidocaine and ketamine combination to ITM would decrease the cumulative opioid consumption. Based on our prior abdominal surgery experience (mean 24 hour morphine use $42.3 \pm 30.5 \mathrm{mg}$ ), we calculated the sample size of 21 subjects per group for an alpha error of 0.05 and power (1-beta) of 0.90 to detect a clinically significant reduction in morphine equivalent consumption of $30 \%$ in first 24 hour and allowing 6 pair-wise comparisons [6]. We added 10 subjects per group to allow for loss to follow-up.

\subsection{Statistical Analysis}

All data were stored in a Microsoft Excel 2017 (Microsoft Corp, Redmond, WA, USA) and then analysed using SPSS version 23 (SPSS Inc., Chicago IL, 2015). The data obtained were compared statistically using repeated-measures analysis of variance (ANOVA) and the post hoc Tukey-HSD test; nonparametric data were evaluated using the Kruskal-Wallis test. $\mathrm{P}<0.05$ was considered to be statistically significant. Descriptive statistics for demographic and baseline characteristics by treatment assignment were provided. For comparing proportions, categorical variables and dichotomous outcomes were analysed using chi-square test and normally distributed continuous repeated measures were analysed using ANOVA. For characteristics possibly associated with endpoints and where clinically important differences were observed, in-between group comparisons of opioid use, pain score and other ordinal endpoints were performed using Tukey's method of multiple comparisons. Probability values less than 0.05 were 
considered statistically significant.

Data are described as mean (standard deviation) (SD) or numbers with percentages. We analysed with a modified intention-to-treat (ITT) strategy. Only those participants who received intervention were included in the analysis. Therefore, those who did not receive ITM were excluded from the data. If there was missing data, it was completed using a last observation carried forward paradigm [7].

\section{Results}

Two hundred and five adult patients scheduled for open major liver resection were assessed for eligibility, 134 met the criteria and 124 were assigned randomization, 31 in each group (Figure 2). A total of four were withdrawn after recruitment as ITM could not be placed. No data could be collected therefore seven were withdrawn from the final analysis as they either remained intubated postoperatively, were re-operated within 24 hours or did not undergo the planned surgery. The final analysis included 27 in the lidocaine, 28 in the ketamine, 29 in the combination and 29 in the placebo group.

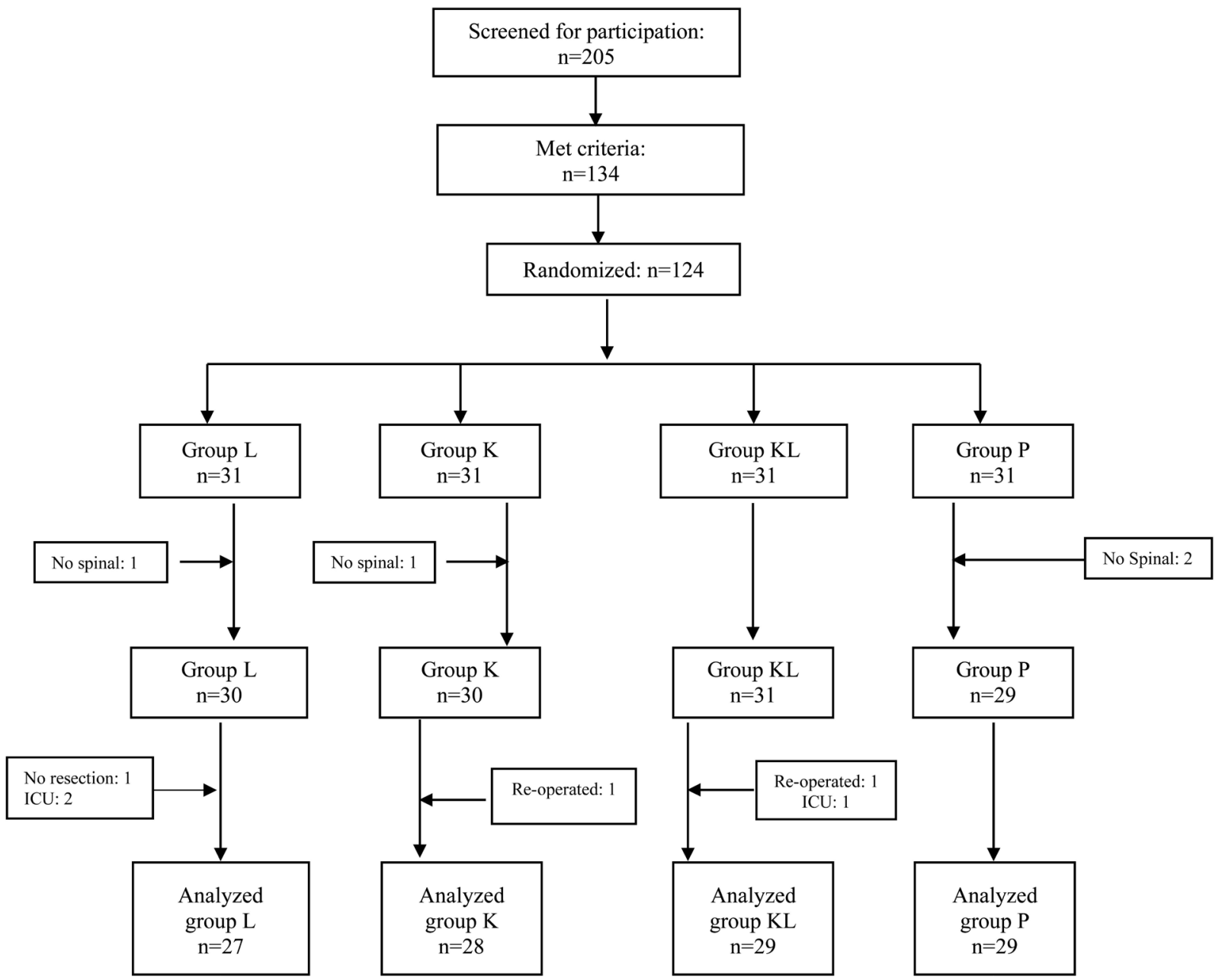

Figure 2. Consort diagram for participants in the study. 


\subsection{Results}

Baseline demographics and surgical procedure duration were comparable between the four groups (Table 1). The primary outcome measure was opioid use (in intravenous morphine equivalent doses) at 24 hours. There was no difference in this outcome, between the groups (group L: $42 \mathrm{mg}$, group K: $35.6 \mathrm{mg}$, group KL: $47.5 \mathrm{mg}$, group P: $34.4 \mathrm{mg} ; \mathrm{p}=0.44$ ). Similarly, no difference was seen at 48 hours when compared to placebo (Table 2 and Table 3 ).

The 24 hours pain scores at rest and movement/deep breathing were similar between the groups (Table 4). At 48 hours, rest pain scores were significantly different between the groups ( $\mathrm{p}=0.03$ ) but there was no difference in pain with movement/deep breathing $(p=0.07)$. On further analysis of the 48-hour data and using a 6-way pairwise comparison, pain at rest in lidocaine group was worse than ketamine and placebo (Table 5). There was no statistically significant difference between the groups at other time points.

Table 1. Characteristics of patients receiving Lidocaine, Ketamine, Lidocaine + Ketamine or Placebo for liver resection. Values are reported as mean (SD) or number.

\begin{tabular}{ccccc}
\hline & $\begin{array}{c}\text { Group L* } \\
(\mathbf{n}=27)\end{array}$ & $\begin{array}{c}\text { Group K}^{\dagger} \\
(\mathbf{n}=28)\end{array}$ & $\begin{array}{c}\text { Group KL } \\
(\mathbf{n}=29)\end{array}$ & $\begin{array}{c}\text { Group P }^{\S} \\
(\mathbf{n}=29)\end{array}$ \\
\hline Sex-M:F & $15: 12$ & $20: 8$ & $18: 11$ & $18: 11$ \\
ASA-PS & 2 & 2 & 1 & 0 \\
2 & 17 & 22 & 20 & 22 \\
3 & 3 & 4 & 8 & 9 \\
4 & $59.7(12.2)$ & $63.3(11.1)$ & $61.1(12.8)$ & $60.1(10.4)$ \\
Age, mean (SD) years & $77.1(16.6)$ & $82.0(18.9)$ & $83.0(19.2)$ & $79.9(19.5)$ \\
Weight, mean (SD) kg & $167.7(11.6)$ & $172.1(9.9)$ & $173.2(8.8)$ & $169.5(11.0)$ \\
Height, mean (SD) cm & $27.4(5.4)$ & $27.7(6.0)$ & $27.5(5.5)$ & $27.6(4.9)$ \\
BMI, mean (SD) kg.m ${ }^{-2}$ & $325.2(94.1)$ & $356.1(99.3)$ & $374.1(138.5)$ & $357.9(116.1)$ \\
Surgical time (min) & & & & \\
\hline
\end{tabular}

${ }^{*}$ group lidocaine, ${ }^{\dagger}$ group ketamine, ${ }^{\ddagger}$ group ketamine + lidocaine, 'group placebo; Abbreviations: ASA: American Society of Anesthesiologists Physical Status.

Table 2. Opioid requirements: morphine equivalents in patients receiving Lidocaine, Ketamine, Lidocaine + Ketamine or Placebo for liver resection. Values are reported as mean (SD) mg/day.

\begin{tabular}{cccccc}
\hline & $\begin{array}{c}\text { Group L* } \\
(\mathbf{n}=27)\end{array}$ & $\begin{array}{c}\text { Group K} \\
(\mathbf{n}=28)\end{array}$ & $\begin{array}{c}\text { Group KL } \\
(\mathbf{n}=29)\end{array}$ & $\begin{array}{c}\text { Group } P^{\S} \\
(\mathbf{n}=29)\end{array}$ & P value \\
\hline 24-hour & $42(26.5)$ & $35.6(32.6)$ & $47.5(44.7)$ & $34.4(30.1)$ & 0.244 \\
48-hour & $37.2(36.1)$ & $35.0(29.0)$ & $38.4(31.1)$ & $41.4(36.5)$ & 0.610 \\
72 -hour & $23.7(26.2)$ & $20(21.5)$ & $27.9(28.8)$ & $28.2(36.3)$ & 0.075 \\
Cumulative & $102.9(65.1)$ & $90.6(68.6)$ & $113.8(89.2)$ & $104.0(79.0)$ & 0.299 \\
\hline
\end{tabular}

${ }^{*}$ group lidocaine, ${ }^{\dagger}$ group ketamine, ${ }^{\ddagger}$ group ketamine + lidocaine, ${ }^{\varsigma}$ group placebo. 
Table 3. Pairwise comparisons for opioid requirements: morphine equivalents in patients receiving Lidocaine, Ketamine, Lidocaine + Ketamine or Placebo for liver resection.

\begin{tabular}{|c|c|c|c|c|c|}
\hline Pain at rest & Groups & Mean difference & Standard error & $95 \% \mathrm{CI}$ & $P$ value \\
\hline \multicolumn{6}{|l|}{ 24-hour } \\
\hline \multirow[t]{3}{*}{$L^{*}$} & $\mathrm{~K}^{\dagger}$ & 6.40 & 9.28 & $-17.82,30.62$ & 0.90 \\
\hline & $\mathrm{KL}^{\ddagger}$ & -5.54 & 9.20 & $-29.56,18.47$ & 0.93 \\
\hline & $\mathrm{P}^{\S}$ & 7.57 & 9.20 & $-16.45,31.47$ & 0.84 \\
\hline \multirow[t]{2}{*}{$\mathrm{K}$} & $\mathrm{KL}$ & -11.94 & 9.12 & $-35.73,11.85$ & 0.56 \\
\hline & $\mathrm{P}$ & 1.16 & 9.12 & $-22.63,24.95$ & 0.99 \\
\hline $\mathrm{KL}$ & $\mathrm{P}$ & 13.11 & 9.04 & $-10.47,36.69$ & 0.47 \\
\hline \multicolumn{6}{|l|}{ 48-hour } \\
\hline \multirow[t]{3}{*}{$\mathrm{L}$} & $\mathrm{K}$ & 2.22 & 8.99 & $-21.23,25.67$ & 0.99 \\
\hline & $\mathrm{KL}$ & -1.22 & 8.91 & $-24.48,22.02$ & 0.99 \\
\hline & $\mathrm{P}$ & -4.23 & 8.91 & $-27.48,19.02$ & 0.96 \\
\hline \multirow[t]{2}{*}{$\mathrm{K}$} & $\mathrm{KL}$ & -3.45 & 8.83 & $-26.48,19.59$ & 0.98 \\
\hline & $\mathrm{P}$ & -6.45 & 8.83 & $-29.48,16.58$ & 0.89 \\
\hline $\mathrm{KL}$ & $\mathrm{P}$ & -3.01 & 8.75 & $-25.84,19.82$ & 0.99 \\
\hline \multicolumn{6}{|l|}{ 72-hour } \\
\hline \multirow[t]{3}{*}{$\mathrm{L}$} & $\mathrm{K}$ & 3.74 & 7.77 & $-16.54,24.01$ & 0.96 \\
\hline & $\mathrm{KL}$ & -4.13 & 7.70 & $-24.23,15.97$ & 0.95 \\
\hline & $\mathrm{P}$ & -4.44 & 7.70 & $-24.54,15.65$ & 0.94 \\
\hline \multirow[t]{2}{*}{$\mathrm{K}$} & $\mathrm{KL}$ & -7.86 & 7.63 & $-27.77,12.05$ & 0.73 \\
\hline & $\mathrm{P}$ & -8.18 & 7.63 & $-28.09,11.73$ & 0.71 \\
\hline $\mathrm{KL}$ & $\mathrm{P}$ & -3.16 & 7.56 & $-20.05,19.42$ & 0.99 \\
\hline \multicolumn{6}{|l|}{ Total use } \\
\hline \multirow[t]{3}{*}{$\mathrm{L}$} & $\mathrm{K}$ & 12.35 & 21.49 & $-43.71,68.42$ & 0.94 \\
\hline & $\mathrm{KL}$ & -10.90 & 21.30 & $-66.48,44.69$ & 0.96 \\
\hline & $\mathrm{P}$ & -1.11 & 21.30 & $-56.70,54.47$ & 0.92 \\
\hline \multirow[t]{2}{*}{$\mathrm{K}$} & $\mathrm{KL}$ & -23.25 & 21.11 & $-78.32,31.82$ & 0.69 \\
\hline & $\mathrm{P}$ & -13.47 & 21.11 & $-68.53,41.60$ & 0.92 \\
\hline $\mathrm{KL}$ & $\mathrm{P}$ & 9.79 & 20.92 & $-44.80,64.37$ & 0.97 \\
\hline
\end{tabular}

* group lidocaine, ${ }^{\dagger}$ group ketamine, ${ }^{\ddagger}$ group ketamine + lidocaine, 'sroup placebo; CI \& P values are corrected with Tukey's HSD (honestly significant difference).

Satisfaction with pain control at 48 hours was significantly higher in the ketamine group $(\mathrm{p}=0.02)$ (Table 4$)$. At all other time points, the difference was not significant. Drug related secondary outcomes were not different between the groups (Table 6). Three patients in lidocaine group and one in the combination group had persistent post-surgical pain at 12 weeks but the numbers were considered too small for any analysis. 
Table 4. Pain scores and satisfaction in patients receiving Lidocaine, Ketamine, Lidocaine + Ketamine or Placebo for liver resection. Data are presented as means (SD).

\begin{tabular}{|c|c|c|c|c|c|c|c|c|c|c|}
\hline & & $\begin{array}{l}\text { Group } L^{*} \\
(n=27)\end{array}$ & $95 \% \mathrm{CI}$ & $\begin{array}{c}\text { Group } K^{\dagger} \\
(n=28)\end{array}$ & $95 \% \mathrm{CI}$ & $\begin{array}{l}\text { Group } \mathrm{KL}^{\ddagger} \\
(\mathrm{n}=29)\end{array}$ & $95 \% \mathrm{CI}$ & $\begin{array}{c}\text { Group } P^{\S} \\
(n=29)\end{array}$ & $95 \% \mathrm{CI}$ & $P$ value \\
\hline \multicolumn{11}{|c|}{ Pain severity (VRS $9-10$ scale) } \\
\hline \multirow{2}{*}{ POD1 } & Rest & $2.7(1.8)$ & $2.04,3.44$ & $2.4(1.9)$ & $1.61,3.16$ & $2.8(2.2)$ & $1.97,3.62$ & $2.8(2.0)$ & $1.99,3.58$ & 0.84 \\
\hline & Movement & $5.7(2.1)$ & $4.85,6.48$ & $5.6(2.5)$ & $4.62,6.61$ & $5.2(2.6)$ & $4.17,6.17$ & $5.0(2.5)$ & $4.17,6.17$ & 0.76 \\
\hline \multirow{2}{*}{ POD 2} & Rest & $3.1(2.3)$ & $2.20,4.02$ & $1.6(1.8)$ & $0.91,2.32$ & $2.3(2.4)$ & $1.41,3.21$ & $1.8(1.4)$ & $1.21,2.29$ & $0.03^{\#}$ \\
\hline & Movement & $5.2(1.9)$ & $4.43,5.94$ & $3.7(2.0)$ & $2.88,4.51$ & $4.9(2.5)$ & $3.95,5.84$ & $5.0(2.2)$ & $4.20,5.87$ & 0.07 \\
\hline \multirow{2}{*}{ POD 3} & Rest & $2.0(1.9)$ & $1.27,2.80$ & $1.6(1.6)$ & $0.97,2.24$ & $2.2(2.3)$ & $1.30,3.05$ & $1.3(1.1)$ & $0.89,1.76$ & 0.27 \\
\hline & Movement & $4.2(1.7)$ & $3.47,4.82$ & $3.5(1.5)$ & $2.84,4.08$ & $4.6(2.7)$ & $3.60,5.64$ & $3.9(1.5)$ & $3.28,4.43$ & 0.34 \\
\hline \multirow{2}{*}{ Wk 6} & Rest & $0.8(1.6)$ & $0.17,1.45$ & $0.2(0.7)$ & $-0.05,0.48$ & $0.8(1.9)$ & $0.09,1.56$ & $0.4(0.6)$ & $0.18,0.67$ & 0.25 \\
\hline & Movement & $1.7(1.7)$ & $1.06,2.42$ & $1.2(2.1)$ & $0.36,2.01$ & $1.9(2.3)$ & $1.01,2.78$ & $1.2(1.3)$ & $0.68,1.67$ & 0.36 \\
\hline \multirow{2}{*}{ Wk 12} & Rest & $0.6(1.5)$ & $0.00,1.19$ & $0.3(0.9)$ & $-0.08,0.65$ & $1.0(2.5)$ & $0.04,1.96$ & $0.3(0.7)$ & $0.04,0.60$ & 0.31 \\
\hline & Movement & $1.2(1.8)$ & $0.52,1.93$ & $0.7(1.8)$ & $0.05,1.43$ & $1.5(2.4)$ & $0.60,2.43$ & $0.9(1.3)$ & $0.34,1.37$ & 0.39 \\
\hline \multicolumn{11}{|c|}{ Satisfaction with pain management (VRS $0-10$ scale) } \\
\hline Day 1 & & $6.6(2.3)$ & $5.70,7.56$ & $7.6(2.6)$ & $6.53,8.57$ & $7.6(2.6)$ & $7.24,8.59$ & $7.1(2.6)$ & $6.09,8.05$ & 0.12 \\
\hline Day 2 & & $6.5(2.4)$ & $5.54,7.42$ & $7.9(2.8)$ & $6.85,9.00$ & $7.6(2.1)$ & $6.73,8.37$ & $6.8(2.7)$ & $5.82,7.84$ & $0.02^{\#}$ \\
\hline Day 3 & & $7.0(2.4)$ & $6.09,7.99$ & $7.7(2.8)$ & $6.61,8.75$ & $7.3(2.4)$ & $6.37,8.18$ & $6.7(2.9)$ & $5.54,7.77$ & 0.33 \\
\hline
\end{tabular}

${ }^{*}$ group lidocaine, ${ }^{\dagger}$ group ketamine, ${ }^{*}$ group ketamine + lidocaine, ${ }^{5}$ group placebo, \#statistically significant; Abbreviations: ${ }^{*}$ POD: postoperative day, ${ }^{\psi} \mathrm{Wk}$ : week, "VRS: verbal rating scale.

Table 5. Pairwise comparisons for pain on day 2 in patients receiving Lidocaine, Ketamine, Lidocaine + Ketamine or Placebo for liver resection.

\begin{tabular}{cccccc}
\hline Pain at rest & Groups & Mean difference & Standard error & $95 \%$ CI & P value \\
\hline $\mathrm{L}^{*}$ & $\mathrm{~K} \dagger$ & 1.43 & 0.54 & $0.04,2.84$ & $0.046^{\#}$ \\
& $\mathrm{KL} \neq$ & 0.80 & 0.54 & $-0.71,2.09$ & 0.446 \\
& $\mathrm{P} \S$ & 1.42 & 0.54 & $0.11,2.84$ & $0.045^{\#}$ \\
$\mathrm{~K}$ & $\mathrm{KL}$ & -0.63 & 0.53 & $-2.14,0.64$ & 0.636 \\
& $\mathrm{P}$ & -0.11 & 0.53 & $-1.32,1.39$ & 0.99 \\
$\mathrm{KL}$ & $\mathrm{P}$ & 0.62 & 0.53 & $-0.57,2.14$ & 0.642 \\
& & $\mathrm{Pain}$ with movement & & \\
$\mathrm{L}$ & $\mathrm{K}$ & 1.36 & 0.58 & $-0.14,2.87$ & 0.093 \\
& $\mathrm{KL}$ & 0.29 & 0.58 & $-1.32,1.69$ & 0.958 \\
& $\mathrm{P}$ & 0.082 & 0.58 & $-1.29,1.66$ & 0.999 \\
$\mathrm{~K}$ & $\mathrm{KL}$ & -1.08 & 0.57 & $-2.67,0.31$ & 0.239 \\
& $\mathrm{P}$ & -1.28 & 0.57 & $-2.63,0.28$ & 0.116 \\
$\mathrm{KL}$ & $\mathrm{P}$ & -0.21 & 0.56 & $2.14,1.46$ & 0.983 \\
\hline
\end{tabular}

* group lidocaine, ${ }^{\dagger}$ group ketamine, " group ketamine + lidocaine, 'group placebo, "statistically significant; CI \& P values are corrected using Tukey's HSD. 
Table 6. Evaluation of side effects related to ketamine, lidocaine or intrathecal morphine in patients receiving Lidocaine, Ketamine, Lidocaine + Ketamine or Placebo for liver resection. Values are expressed as means (SD), number or \% as indicated.

\begin{tabular}{cccccccccc}
\hline & \multicolumn{2}{c}{ Group $L^{*}(\mathrm{n}=27)$} & \multicolumn{2}{c}{ Group $\mathrm{K}^{\dagger}(\mathrm{n}=\mathbf{2 8})$} & \multicolumn{2}{c}{ Group KL $(\mathrm{n}=29)$} & \multicolumn{2}{c}{ Group $\mathrm{P}^{\S}(\mathrm{n}=29)$} & $\mathrm{P}_{\text {value }}$ \\
\hline $\mathrm{N} / \mathrm{V}^{\sharp}$ & $0.6(0.9)$ & $10(37 \%)$ & $0.9(2.0)$ & $10(35.7 \%)$ & $0.3(0.6)$ & $8(27.6 \%)$ & $0.9(1.1)$ & $14(48.3 \%)$ & 0.72 \\
Pruritus & $0.8(1.1)$ & $10(37 \%)$ & $0.9(1.2)$ & $13(46.4 \%)$ & $0.7(1.0)$ & $11(37.9 \%)$ & $0.8(0.8)$ & $16(55.2 \%)$ & 0.12 \\
Lightheadedness & $0.4(0.6)$ & $10(37 \%)$ & $0.5(0.8)$ & $11(39.3 \%)$ & $0.7(0.9)$ & $13(44.8 \%)$ & $0.6(0.6)$ & $14(48.3 \%)$ & 0.73 \\
Oral numbness & $0.0(0.2)$ & $1(3.7 \%)$ & $0.1(0.4)$ & $2(7.1 \%)$ & $0.1(0.3)$ & $2(6.9 \%)$ & $0.0(0.0)$ & $0(0 \%)$ & 0.53 \\
Hallucinations & $0.4(0.7)$ & $7(25.9 \%)$ & $0.3(0.6)$ & $6(21.4 \%)$ & $0.5(0.8)$ & $10(34.5 \%)$ & $0.4(0.9)$ & $6(20.7 \%)$ & 0.85 \\
\hline
\end{tabular}

${ }^{*}$ group lidocaine, ${ }^{\dagger}$ group ketamine, ${ }^{*}$ group ketamine + lidocaine, ${ }^{5}$ group placebo, Abbreviations: ${ }^{\sharp}$ nausea/vomiting.

\subsection{Discussion}

This study assessed the influence of intraoperative low dose ketamine, lidocaine and their combination on postoperative pain in the presence of ITM. As lidocaine and ketamine are known to improve analgesia, it was hypothesized that the combination of both drugs would improve analgesia further and reduce postoperative opioid use. Our main results show that there was no incremental benefit with either drug or its combination on postoperative opioid use with an anaesthetic regimen using ITM. All groups used similar doses opioid doses, achieved similar pain score and analgesia on POD1.

Though it is a classic local anaesthetic, systemic lidocaine has analgesic, antinociceptive, immuno-modulating, and anti-inflammatory properties [8] [9]. Several studies have shown reduction in postoperative opioid consumption, pain score, fatigue score, length of hospitalization and improved bowel function whereas others have failed to show a benefit [10] [11]. Several meta-analyses have examined the effects of perioperative lidocaine though some have questions on the usefulness of the technique [12] [13]. Khan and colleagues showed that continuing lidocaine beyond 60 minutes after surgery did not have any analgesic or gastrointestinal benefits [14]. The results of a recent Cochrane review were inconclusive regarding the benefits of lidocaine on pain scores and opioid consumption when compared to placebo [4].

Ketamine is a complicated anaesthetic drug with anti-inflammatory, antinociceptive and anti-hyperalgesic properties with ability to reverse surgically induced enhanced pain sensitivity [15]. Similar to lidocaine, randomized studies have demonstrated inconsistent results with reduced opioid consumption and attenuation of the secretion of pro-inflammatory markers in some studies but not in others [16] [17] [18]. In our study, ketamine failed to reduce opioid consumption on POD1, the primary outcome measure.

The clinical consequences of the combination and the doses of both drugs are unknown as there are not many studies. The trial by Grady et al. was stopped midway as both lidocaine, ketamine and combination crossed the futility boundary [11]. Most widely accepted doses for perioperative lidocaine infusion are $0.5-3 \mathrm{mg} / \mathrm{kg} / \mathrm{hr}$ [19]. For ketamine, the recommended doses are $0.1-0.6$ $\mathrm{mg} / \mathrm{kg} / \mathrm{hr}$ though a maximum of $1 \mathrm{mg} / \mathrm{kg} / \mathrm{hr}$ can be used [20]. We chose smaller 
than recommended doses of both drugs as they are metabolized in liver exclusively and we speculated that liver resection could reduce drug clearance through the physical reduction in the liver parenchyma as well as the surgically induced transient inefficiency or the remaining organ [21] [22]. Indications for surgery in this study were liver resection due to malignancy and therefore associated long standing liver dysfunction was unlikely. With high heterogeneity among studies, optimal dose and the correct regimen have not been determined and similar, higher or lower doses with or without boluses have been used [23].

ITM has been used since the beginning of the $20^{\text {th }}$ century and is a popular technique used to provide postoperative analgesia. It is safe, effective and has shown to decrease postoperative opioid requirement in major abdominal surgeries including liver resection with no major side effects [2]. It produces intense analgesia for up to 24 hours [24]. However, ITM may be associated with adverse side-effects though the incidence was infrequent in the present study (Table 6). For major abdominal surgeries, the optimal dose of ITM remains unknown. Doses as low as $0.05 \mathrm{mg}$ and as high as $1 \mathrm{mg}$ have been used to provide good postoperative analgesia [25]. As far as we know, there are no studies comparing the impact of intravenous infusion of lidocaine and ketamine on postoperative opioid consumption and pain control in the milieu of ITM after major liver resection.

We had several thought-provoking findings in secondary outcomes. These findings suggested improved analgesia and patient satisfaction with intraoperative ketamine on POD2. Though this was not the primary objective of our study, at 48 -hour we found significantly better pain scores in the ketamine group at rest ( $\mathrm{p}=0.028)$ but not with movement $(\mathrm{p}=0.07)$. On comparing all possible pair means, lidocaine was significantly worse that ketamine and even placebo (lidocaine vs ketamine $\mathrm{p}=0.046$, lidocaine vs placebo $\mathrm{p}=0.045$, lidocaine vs combination $\mathrm{p}=0.446$ ). The ketamine-lidocaine combination did not offer any significant advantage at any time points. We did not observe any psycho-active side effects of ketamine.

All analyses beyond the primary outcome of 24 hours opioid consumption were secondary and should be interpreted with caution as the study was not powered for these outcomes. From a statistical standpoint, there will always be concerns whether the analyses are reported with or without correction. If the secondary analyses are reported with unadjusted $\mathrm{p}$ values and no correction is applied, it alters the chances of finding statistical significance [26]. However, correction for multiple comparisons carries a risk of increasing false negatives. Tukey's HSD compares all possible pairwise combinations of means with appropriate adjustment for the multiple testing.

\subsection{Limitations}

Our study had several limitations. First, we used lower than recommended doses of lidocaine and ketamine. It is possible that our results may have been different 
if the infusions were of higher concentration, longer duration or both. Second, we did not look at functional outcomes like gastrointestinal recovery and length of stay that may have reflected reduction in the opioid burden and the time to discharge. Third, the sample size estimation was based on our experience on opioid consumption in an abdominal surgery model as there were not many studies available with a similar design. It is possible that the study may have been underpowered due to the effect size of $30 \%$ being too large in the setting of ITM, variation from the standard deviation of the primary outcome used in the sample size calculation or any other known/unknown factor. Fourth, we had failed lumber puncture in 4 patients (3\%). Quoted failure rates are between $1 \%-4 \%$ [27]. It is possible that another person's attempt may have been successful. Another important limitation was that protocols for expedited recovery after surgery (ERAS) were introduced at our institution during the course of the study. It is well known that ERAS impacts the postoperative pain intensity, and this may have influenced the study outcomes.

This study was not powered to look at the secondary outcomes therefore we need to interpret the results with discretion. Though it is difficult to apply these results to all patient population, these findings do warrant further studies and can be considered in developing analgesic strategies particularly where supplies may be limited.

\section{Conclusion}

Our results showed that intraoperative infusion of low dose ketamine, lidocaine or their combination with ITM and multimodal analgesia in the background, did not provide additional analgesia or reduce the 24 hours opioid use. Low dose ketamine showed advantage in terms of better pain scores and patient satisfaction at 48 hours when used with ITM but without a reduction in opioid use. Low dose lidocaine with or without ketamine was similar to placebo and did not offer any advantage over ITM following major open liver resection.

\section{Acknowledgements}

This work received the best paper prize at the 2018 Canadian Anesthesia Society annual meeting in Montreal, Quebec in June 2018. No competing interests or funding.

\section{Conflicts of Interest}

The authors declare no conflicts of interest regarding the publication of this paper.

\section{References}

[1] Shontz, R., Karuparthy, V., Temple, R. and Brennan, T.J. (2009) Prevalence and Risk Factors Predisposing to Coagulopathy in Patients Receiving Epidural Analgesia for Hepatic Surgery. Regional Anesthesia and Pain Medicine, 34, 308-311. https://doi.org/10.1097/AAP.0b013e3181ac7d00 
[2] Roy, J.D., Massicotte, L., Sassine, M.P., Seal, R.F. and Roy, A. (2006) A Comparison of Intrathecal Morphine/Fentanyl and Patient-Controlled Pnalgesia with $\mathrm{Pa}$ tient-Controlled Analgesia Alone for Analgesia after Liver Resection. Anesthesia \& Analgesia, 103, 990-994. https://doi.org/10.1213/01.ane.0000238040.41872.7e

[3] Chou, R., Gordon, D.B., de Leon-Casasola, O.A., Rosenberg, J.M., Bickler, S., Brennan, T., et al. (2016) Management of Postoperative Pain: A Clinical Practice Guideline From the American Pain Society, the American Society of Regional Anesthesia and Pain Medicine, and the American Society of Anesthesiologists' Committee on Regional Anesthesia, Executive Committee, and Administrative Council. Journal of Pain, 17, 131-157. https://doi.org/10.1016/j.jpain.2015.12.008

[4] Weibel, S., Jelting, Y., Pace, N.L., Helf, A., Eberhart, L.H., Hahnenkamp, K., et al. (2018) Continuous Intravenous Perioperative Lidocaine Infusion for Postoperative Pain and Recovery in Adults. Cochrane Database Systematic Review, 6, Article No. CD009642. https://doi.org/10.1002/14651858.CD009642.pub3

[5] Laskowski, K., Stirling, A., McCay, W.P. and Lim, H.J. (2011) A Systematic Review of Intravenous Ketamine for Postoperative Analgesia. Canadian Journal of Anesthesia, 58, 911-923. https://doi.org/10.1007/s12630-011-9560-0

[6] Chow, S., Shao, J. and Wang, H. (2008) Sample Size Calculations in Clinical Research. 2nd Edition, Chapman and Hall/CRC Taylor and Francis Group, Florida.

[7] Woolard, R.H., Carty, K., Wirtz, P., Longabaugh, R., Nirenberg, T.D., Minugh, P.A., et al. (2004) Research Fundamentals: Follow-Up of Subjects in Clinical Trials: Addressing Subject Attrition. Academic Emergency Medicine, 11, 859-866. https://doi.org/10.1197/j.aem.2003.09.021

[8] Estebe, J.P. (2017) Intravenous Lidocaine. Best Practice and Research: Clinical Anaesthesiology, 31, 513-521. https://doi.org/10.1016/j.bpa.2017.05.005

[9] Kundra, P. and Vinayagam, S. (2020) Perioperative Intravenous Lidocaine: Crossing Local Boundaries and Reaching Systemic Horizons. Indian Journal of Anesthesia, 64, 363-365.

[10] Vigneault, L., Turgeon, A.F., Cote, D., Lauzier, F., Zarychanski, R., Moore, L., et al. (2011) Perioperative Intravenous Lidocaine Infusion for Postoperative Pain Control: A Meta-Analysis of Randomized Controlled Trials. Canadian Journal of Anesthesia, 58, 22-37. https://doi.org/10.1007/s12630-010-9407-0

[11] Grady, M.V., Mascha, E., Sessler, D.I. and Kurz, A. (2012) The Effect of Perioperative Intravenous Lidocaine and Ketamine on Recovery after Abdominal Hysterectomy. Anesthesia \& Analgesia, 115, 1078-1084. https://doi.org/10.1213/ANE.0b013e3182662e01

[12] Marret, E., Rolin, M., Beaussier, M. and Bonnet, F. (2008) Meta-Analysis of Intravenous Lidocaine and Postoperative Recovery after Abdominal Surgery. British Journal of Surgery, 95, 1331-1338. https://doi.org/10.1002/bjs.6375

[13] Weibel, S., Jokinen, J., Pace, N.L, Schnabel, A., Hollman, M.W., Hanenkamp, K., et al. (2016) Efficacy and Safety of Intravenous Lidocaine for Postoperative Analgesia and Recovery after Surgery: A Systematic Review with Trial Sequential Analysis. British Journal of Anesthesia, 116, 770-783. https://doi.org/10.1093/bja/aew101

[14] Khan, J.S., Yousuf, M., Victor, J.C., Sharma, A. and Siddiqui, N. (2016) An Estimation for an Appropriate End Time for an Intraoperative Intravenous Lidocaine Infusion in Bowel Surgery: A Comparative Meta-Analysis. Journal of Clinical Anesthesia, 28, 95-104. https://doi.org/10.1016/j.jclinane.2015.07.007

[15] Gorlin, A.W., Rosenfeld, D.M. and Ramakrishna, H. (2016) Intravenous Sub-Anesthetic Ketamine for Perioperative Analgesia. Journal of Anaesthesiology and Clinical Phar- 
macology, 32, 160-167. https://doi.org/10.4103/0970-9185.182085

[16] Zakine, J., Samarcq, D., Lorne, E., Moubarak, M., Montravers, P., Beloucif, S., et al. (2008) Postoperative Ketamine Administration Decreases Morphine Consumption in Major Abdominal Surgery: A Prospective, Randomized, Double-Blind, Controlled Study. Anesthesia \& Analgesia, 106, 1856-1861.

https://doi.org/10.1213/ane.0b013e3181732776

[17] D'Alonzo, R.C., Bennett-Guerrero, E., Podgoreanu, M., D'Amico, T.A., Harpole, D.H. and Shaw, A.D. (2011) A Randomized, Double Blind, Placebo Controlled Clinical Trial of The Preoperative Use of Ketamine for Reducing Inflammation and Pain after Thoracic Surgery. Journal of Anesthesia, 25, 672-678. https://doi.org/10.1007/s00540-011-1206-4

[18] Ibrahim, T.H., Abdelrahman, H.S., Alharbi, M.A., Zabani, I.A., Ismail, M.F. and Kary, H. (2017) Effect of Ketamine on Pro- and Anti-Inflammatory Cytokine Response in Paediatric Cardiac Surgery: A Prospective Randomised Controlled Study. Indian Journal of Anesthesia, 61, 549-555. https://doi.org/10.4103/ija.IJA $607 \quad 16$

[19] Eipe, N., Gupta, S. and Penning, J. (2016) Intravenous lidocaine for Acute Pain: An Evidence-Based Clinical Update. British Journal of Anesthesia Education, 16, 292-298. https://doi.org/10.1093/bjaed/mkw008

[20] Himmelseher, S. and Durieux, M.E. (2005) Ketamine for Perioperative Pain Management. Anesthesiology, 102, 211-220. https://doi.org/10.1097/00000542-200501000-00030

[21] Chang, T. and Glazko, A.J. (1974) Biotransformation and Disposition of Ketamine. International Anesthesiology Clinics, 12, 157-177. https://doi.org/10.1097/00004311-197412020-00018

[22] Orlando, R., Piccoli, P., De Martin, S., Padrini, R., Floreani, M. and Palatini, P. (2004) Cytochrome P450 1A2 Is a Major Determinant of Lidocaine Metabolism in Vivo: Effects of Liver Function. Clinical Pharmacology and Therapeutics, 75, 80-88. https://doi.org/10.1016/j.clpt.2003.09.007

[23] Jouguelet-Lacoste, J., La Colla, L., Schilling, D. and Chelly, J.E. (2015) The Use of Intravenous Infusion or Single Dose of Low-Dose Ketamine for Postoperative Analgesia: A Review of the Current Literature. Pain Medicine, 16, 383-403. https://doi.org/10.1111/pme.12619

[24] Bujedo, B. (2012) A Clinical Approach to Neuraxial Morphine for the Treatment of Postoperative Pain. Pain Research and Treatment, 2012, Article ID: 612145. https://doi.org/10.1155/2012/612145

[25] Fares, K.M., Mohamed, S.A. and Abdel-Ghaffar, H.S. (2014) High Dose Intrathecal Morphine for Major Abdominal Cancer Surgery: A Prospective Double-Blind, Dose-Finding Clinical Study. Pain Physician, 17, 255-264.

[26] Freemantle, N. (2001) Interpreting the Results of Secondary End Points and Subgroup Analyses in Clinical Trials: Should We Lock the Crazy Aunt in the Attic? British Medical Journal, 322, 989-991. https://doi.org/10.1136/bmj.322.7292.989

[27] Fettes, P.D.W., Jansson, J.R. and Wildsmith, J.A.W. (2009) Failed Spinal Anesthesia: Mechanisms, Management, and Prevention. British Journal of Anesthesia, 102, 739-748. https://doi.org/10.1093/bja/aep096 